\title{
RICCI CURVATURE AND EIGENVALUE ESTIMATE ON LOCALLY FINITE GRAPHS
}

\author{
Yong Lin AND SHIng-TUng YAU
}

\begin{abstract}
We give a generalizations of lower Ricci curvature bound in the framework of graphs. We prove that the Ricci curvature in the sense of Bakry and Emery is bounded below by -1 on locally finite graphs. The Ricci flat graph in the sense of Chung and Yau is proved to be a graph with Ricci curvature bounded below by zero. We also get an estimate for the eigenvalue of Laplace operator on finite graphs:

$$
\lambda \geq \frac{1}{d D(\exp (d D+1)-1)},
$$
\end{abstract}

where $d$ is the weighted degree of $G$, and $D$ is the diameter of $G$.

\section{Introduction}

The Ricci curvature plays a very important role on geometric analysis on Riemannian manifolds. Many results are established on manifolds with non-negative Ricci curvature or on manifolds with Ricci curvature bounded below. For analysis on graphs, there is no way to define the derivative, so we can't define the Ricci curvature in classical sense. However, Bakry and Emery find a way to define the "lower Ricci curvature bound" through the heat semigroup $\left(P_{t}\right)_{t \geq 0}$ on a metric measure space $M$. In fact to say that Ricci curvature is bounded below by a constant $-K$ is equivalent to say that

$$
\left[\frac{1}{2}\left(\Delta-\frac{\partial}{\partial t}\right)\right]^{2}\left(P_{t} f\right)^{2} \geq-K\left[\frac{1}{2}\left(\Delta-\frac{\partial}{\partial t}\right)\right]\left(P_{t} f\right)^{2}
$$

for any $f \in C_{0}^{\infty}(M), t \geq 0$. In this setting, Bakry, Ledoux and Qian [2] established several dimension free estimates for logarithmic sobolev constants and constant spectral gap for a diffusion semigroup on a so-called length space. They also proved a dimension-free version of the famous Li-Yau's [7] heat kernel upper bound for the length spaces with non-negative Ricci curvature. We will use the idea of Bakry and Emery [1] to give a notion of a "lower Ricci curvature bound" on graphs and to prove that Ricci curvature on Locally finite graphs is always bounded below by a constant. Recently Sturm [12] has given the definition of a "lower Ricci curvature bound" on metric measure space and prove that it is stable under an appropriate notion of convergence of the spaces. There are some recent works on giving a good notion for a metric measure space to have a "lower Ricci curvature bound", see [8] and [9].

Received by the editors April 3, 2009. Revision received November 22, 2009.

${ }^{0}$ Classification:[2000]31C20, 31C05

The first author is supported by National Natural Science Foundation of China (Grant Nos 10871202)

Keywords: Laplace operator on graphs, Ricci curvature, eigenvalue estimate, analysis on graphs. 
Suppose $G$ is a unordered graph with vertex set $V$ ( $V$ could be an infinite set) and edge set $E$. If for every vertex $x$ of $V$, the number of edges connect to $x$ is finite, we say that $G$ is a locally finite graph. $(x, y)$ denotes the edge of $G$ connected by the two vertices $x$ and $y$. The distance between two vertices is the minimum number of edges to connect them, the diameter of $G$ is the maximum of all the distances of the graph. $\mu_{x y}=\mu_{y x} \geq 0$ is a symmetric weight on $V \times V$. We denote the degree of vertex $X$ by $d_{x}=\sum_{(x, y) \in E} \mu_{x y}$. The weighted degree of the graph $G$ is

$$
d=\sup _{x \in V} \sup _{(x, y) \in E} \frac{d_{x}}{\mu_{x y}} .
$$

Let

$$
V^{R}=\{f \mid f: V \rightarrow R\}
$$

The Laplace operator $\Delta$ of a graph $G$ is

$$
\Delta f(x)=\frac{1}{d_{x}} \sum_{(x, y) \in E} \mu_{x y}[f(y)-f(x)]
$$

for $f \in V^{R}$.

Define a bilinear operator

$$
\Gamma: V^{R} \times V^{R} \rightarrow V^{R}
$$

by

$$
\Gamma(f, g)(x)=\frac{1}{2}\{\Delta(f(x) g(x))-f(x) \Delta g(x)-g(x) \Delta f(x)\} .
$$

According to Bakry and Emery [1], the Ricci curvature operators $\Gamma_{2}$ is defined by iterating the $\Gamma$ :

$$
\Gamma_{2}(f, g)(x)=\frac{1}{2}\{\Delta \Gamma(f, g)(x)-\Gamma(f, \Delta g)(x)-\Gamma(g, \Delta f)(x)\}
$$

For simplicity, we will omit the variable $x$ in the following equations. We should notice that all the equations will hold locally for every $x \in V$.

Definition 1.1. The operator $\Delta$ satisfies the curvature-dimension type inequality $C D(m, K)(m \in(1,+\infty])$ if

$$
\Gamma_{2}(f, f) \geq \frac{1}{m}(\Delta f)^{2}+K \Gamma(f, f) .
$$

We call $m$ the dimension of the operator $\Delta$ and $K$ the lower bound of the Ricci curvature of the operator $\Delta$.

It is easy to see that for $m<m^{\prime}$, the operator $\Delta$ satisfies the curvature-dimension type inequality $C D\left(m^{\prime}, K\right)$ if it satisfies the curvature-dimension type inequality $C D(m, K)$.

If $\Gamma_{2} \geq K \Gamma$, we say that $\Delta$ satisfies $C D(\infty, K)$.

For Laplace operator $\Delta$ on a complete $m$ dimensional Riemannion manifold, the operator $\Delta$ satisfies $C D(m, K)$ iff the Ricci curvature of the Riemanian manifold is bounded below by constant $K$.

In this paper, we will prove the following results. 
Theorem 1.2. Suppose $G$ is a locally finite graph and the weighted degree of the graph $G$ is finite, $\Delta$ is the Laplace operator of $G$. Then we have

$$
\Gamma_{2}(f, f) \geq \frac{1}{2} \cdot(\Delta f)^{2}+\left(\frac{1}{d}-1\right) \Gamma(f, f) .
$$

i.e.the Laplace operator $\Delta$ satisfies $C D\left(2, \frac{1}{d}-1\right)$.

If the weighted degree of graph $G$ is not finite, we have

Theorem 1.3. Suppose $G$ is a locally finite graph, $\Delta$ is the Laplace operator of $G$, then we have

$$
\Gamma_{2}(f, f) \geq \frac{1}{2} \cdot(\Delta f)^{2}-\Gamma(f, f) .
$$

i.e. the Laplace operator $\Delta$ satisfies $C D(2,-1)$.

Bakry, Ledoux and Qian [2] studied this curvature operator $\Gamma_{2}$ on the $\sigma$-finite measure space $(M, B(M), \mu)$, where $M$ is a separable Hausdorff space with its Borel $\sigma$-field $B(M)$ and $\mu$ is a $\sigma$-finite measure on $(M, B(M))$. They gave a $\mu$-symmetric diffusion transition semigroup $\left(P_{t}\right)_{t \geq 0}$, and its Dirichlet form $(\mathcal{E}, F)$ by definition:

$$
\mathcal{E}(f, f)=\lim _{t \rightarrow 0} \frac{1}{t}<f-P_{t} f, f>, f \in L^{2}(M, \mu),
$$

and $F=\left\{f \in L^{2}(M, \mu): \mathcal{E}(f, f)<\infty\right\}$, where $<., .>$ denotes the scale product in $L^{2}(M, \mu)$.

The generator of the semigroup $\left(P_{t}\right)_{t>0}$ is denoted by $\Delta$ with its domain $D(\Delta)$. Assume that there is an algebra $A$ consisting of some continuous functions on $M$; densed both in $C(M)$ and $L^{p}(M, \mu)(p \geq 1)$, and $A \subset D(\Delta)$. Then for the bilinear operator $\Gamma: A \times A \rightarrow A$,

$$
\mathcal{E}(f, f)=\int \Gamma(f, f) d \mu
$$

$\left(P_{t}\right)_{t \geq 0}$ is called a diffusion semigroup, if its generator $\Delta$ satisfies the chain role:

$$
\Delta F(u)=\sum_{i=1}^{m} \frac{\partial F}{\partial x^{i}} \Delta u^{i}+\sum_{i, j=1}^{m} \frac{\partial^{2} F}{\partial x^{i} \partial x^{j}} \Gamma\left(u^{i}, u^{j}\right),
$$

for $u=\left(u^{1}, \ldots, u^{m}\right), u^{i} \in A$ and $F \in C_{b}^{2}\left(R^{m}\right)$.

For the Laplace operator $\Delta$ on graphs, the functions in the domain $D(\Delta)$ are discrete, so it is not a diffusion operator in the above sense. But from the following Lemma, it is a correct operator on the definition of Ricci curvature operator on graphs. In the classic case,

$$
\Gamma(f, f)=|\nabla f|^{2} .
$$

For a graph $G$,

$$
|\nabla f|^{2}(x)=\frac{1}{d_{x}} \sum_{(x, y) \in E} \mu_{x y}[f(x)-f(y)]^{2} .
$$

Lemma 1.4. Suppose that $G$ is a locally finite graph and $\Delta$ is the Laplace operator on $G$, then

$$
\Gamma(f, f)=\frac{1}{2}|\nabla f|^{2}=\frac{1}{2}\left\{\Delta\left(f^{2}\right)-2 f \Delta f\right\}
$$


Proof. From the definition of Laplacian $\Delta$,

$$
\begin{aligned}
\Delta\left(f^{2}(x)\right) & =\frac{1}{d_{x}} \sum_{(x, y) \in E} \mu_{x y}\left[f^{2}(y)-f^{2}(x)\right] \\
& =\frac{1}{d_{x}} \sum_{(x, y) \in E} \mu_{x y}[f(y)-f(x)][f(y)+f(x)] \\
& =\frac{2}{d_{x}} \sum_{(x, y) \in E} \mu_{x y}[f(y)-f(x)] f(x)+\frac{1}{d_{x}} \sum_{(x, y) \in E} \mu_{x y}[f(y)-f(x)]^{2} \\
& =2 f(x) \Delta f(x)+|\nabla f|^{2}(x) .
\end{aligned}
$$

So

$$
\Gamma(f, f)=\frac{1}{2}|\nabla f|^{2}=\frac{1}{2}\left\{\Delta\left(f^{2}\right)-2 f \Delta f\right\} .
$$

After this paper was finished. We notice that Ollivier [10] gave a notion of Ricci curvature valid on arbitrary metric spaces, such as graphs. We can also prove that the Ricci curvature in the sence of Ollivier on graphs also has a lower bound. For Riemannian manifold with a differential operator associated with a stochastic differential equation. The Ricci curvature in the sense of Bakry-Emery is comparitive with the Ricci curvature in the sense of Ollivier, see [10]. However in other case, it is not clear what is the relation between this two Ricci curvature.

Let $(X, d)$ be a Polish metric space, equipped with its Borel $\sigma$-algebra. A random walk $m$ on $X$ is a family of probability measures $m_{x}(\cdot)$ on $X$ for each $x \in X$, satisfying the following two technical assumptions: (i) the measure $m_{x}$ depends measurably on the point $x \in X$; (ii) each measure $m_{x}$ has finite first moment, i.e. for some(hence any) $o \in X$, for any $x \in X$ one has $\int d(o, y) d m_{x}(y)<\infty$.

The Ricci curvature $R(x, y)$ of $(X, d, m)$ along with $(x y)$ defined by Ollivier is:

$$
R(x, y):=1-\frac{\tau_{1}\left(m_{x}, m_{y}\right)}{d(x, y)},
$$

where $\tau_{1}\left(m_{x}, m_{y}\right)=\sup _{f \in \Omega}\left[\int f d m_{x}-\int f d m_{y}\right], \Omega=\left\{f: X \rightarrow \mathbb{R}|| f\left(z_{1}\right)-f\left(z_{2}\right) \mid \leq\right.$ $\left.d\left(z_{1}, z_{2}\right)\right\}$.

Ollivier found that (Proposition 19 in [10]) in geodesic space, it is enough to know $R(x, y)$ for close point, for example, on graphs, we can assume that $x$ is adjacent to $y$.

Let $G$ be an infinite weighted and connected graph(for simplicity, we assume all the weight equal to 1$)$, on which is endowed with a metric $d$ such that $d\left(v_{1}, v_{2}\right)$ is the smallest length of pathes connecting $v_{1}$ and $v_{2}$. We denote $v_{1} \sim v_{2}$ if $v_{1}$ is a neighbor of $v_{2}$.

Suppose $x, y$ are vertices of the graph $G$ with their degree $d_{x}$ and $d_{y}$ respectively. We assume that there is an edge connecting $x$ and $y$.

Under the above assumption, we have the following proposition.

Proposition 1.5. The Ricci curvature of Ollivier $R(x, y) \geq \frac{2}{d_{x}}+\frac{2}{d_{y}}-2$. 
Proof. Let $\Omega=\left\{f: G \rightarrow \mathbb{R}|| f\left(z_{1}\right)-f\left(z_{2}\right) \mid \leq d\left(z_{1}, z_{2}\right)\right\}$.

For any vertex $v$, a probability measure $m_{x}$ related to $v$ is defined by

$$
m_{v}=\frac{1}{d_{v}} \sum_{w \sim v} \delta_{w}
$$

where $d_{v}$ is the degree of $v$ and $\delta_{w}$ is the atomic measure centered at $w$. It follows from [10] that the Ricci curvature

$$
R(x, y)=1-\frac{\sup _{f \in \Omega}\left[\int f d m_{x}-\int f d m_{y}\right]}{d(x, y)} .
$$

Here $d(x, y)=1$. It suffices to show that for any $f \in \Omega$,

$$
\int f d m_{x}-\int f d m_{y} \leq 3-\frac{2}{d_{x}}-\frac{2}{d_{y}} .
$$

Since $f \in \Omega$, we have $\left|f\left(v_{1}\right)-f\left(v_{2}\right)\right| \leq 1$ for any $v_{1} \sim v_{2}$. Hence

$$
\begin{aligned}
& \int f d m_{x}-\int f d m_{y} \\
= & f(x)\left(0-\frac{1}{d_{y}}\right)+f(y)\left(\frac{1}{d_{x}}-0\right)+\frac{1}{d_{x}} \sum_{w \sim x, w \neq y} f(w)-\frac{1}{d_{y}} \sum_{u \sim y, u \neq x} f(u) \\
= & f(x)\left(\frac{d_{x}-1}{d_{x}}-\frac{1}{d_{y}}\right)+f(y)\left(\frac{1}{d_{x}}-\frac{d_{y}-1}{d_{y}}\right) \\
& +\frac{1}{d_{x}} \sum_{w \sim x, w \neq y}[f(w)-f(x)]-\frac{1}{d_{y}} \sum_{u \sim y, u \neq x}[f(u)-f(y)] \\
= & \left(\frac{d_{x} d_{y}-d_{x}-d_{y}}{d_{x} d_{y}}\right)[f(x)-f(y)] \\
& +\frac{1}{d_{x}} \sum_{w \sim x, w \neq y}[f(w)-f(x)]-\frac{1}{d_{y}} \sum_{u \sim y, u \neq x}[f(u)-f(y)] \\
\leq & \left(\frac{d_{x} d_{y}-d_{x}-d_{y}}{d_{x} d_{y}}\right)+\frac{d_{x}-1}{d_{x}}+\frac{d_{y}-1}{d_{y}} \\
= & 3-\frac{2}{d_{x}}-\frac{2}{d_{y}} .
\end{aligned}
$$

That means inequality (1.1) holds and thus Proposition 1.5 is proved.

In [5], Chung and Yau gave a notion of Ricci flat graphs. For a vertex $v$, the neighborhood $N(v)$ of $v$ consists of $v$ and vertices adjacent to $v$. We say graph $G$ has a local $k$-frame at $v$ if there are mappings $\eta_{1}, \ldots, \eta_{k}: N(v) \rightarrow V$ satisfying

(1) $G$ is $k$-regular;

(2) $u$ is adjacent to $\eta_{i} u$ for every $u \in V$ and $1 \leq i \leq k$;

(3) $\eta_{i} u \neq \eta_{j} u$ if $i \neq j$.

A graph $G$ is said to be Ricci flat if $G$ has a local $k$-frame and

$$
\bigcup_{j} \eta_{i} \eta_{j} v=\bigcup_{j} \eta_{j} \eta_{i} v
$$

for any $i$ and vertex $v$. 
For example, the grid $Z^{n}$ is Ricci flat.

We will show that the Ricci flat graphs are graphs with Ricci curvature bounded below by zero. That is

Proposition 1.6. (1) Suppose a graph $G$ is Ricci flat, $\Delta$ is the Laplace operator of $G$, then we have

$$
\Gamma_{2}(f, f) \geq 0 \text {. }
$$

i.e. the Laplace operator $\Delta$ satisfies $C D(\infty, 0)$.

(2)Suppose the graph $G$ is triangle graph with three vertices(a special Ricci flat graph), then we have

$$
\Gamma_{2}(f, f) \geq \frac{1}{2} \cdot(\Delta f)^{2}+\frac{1}{4} \Gamma(f, f) .
$$

i.e. the Laplace operator $\Delta$ satisfies $C D\left(2, \frac{1}{4}\right)$.

We can also show that the Ricci flat graphs are graphs with Ricci curvature bounded below by zero in the sense of Ollivier. In most cases, the Ricci curvature is zero, except for some very special examples like complete graph(every two vertices of graph connected by an eage), the Ricci curvature is positive. The proof is similar to the case of the grid $Z^{n}$, see Example 5 in [10]. See also the proof of Proposition 1.5.

Suppose a function $f: V \rightarrow R$ satisfies

$$
\begin{aligned}
(-\Delta) f(x) & =\frac{1}{d_{x}} \sum_{(x, y) \in E} \mu_{x y}[f(x)-f(y)] \\
& =\lambda f(x),
\end{aligned}
$$
$\lambda$.

then $f$ is called a harmonic eigenfunction of Laplace operator on $G$ with eigenvalue

For Riemannian manifold with lower bound on Ricci curvature, Li and Yau [6] proved the following result by using the gradient estimate of harmonic eigenfunction.

Theorem 1.7. Let $M$ be a compact Riemannian manifold of empty boundary with lower Ricci curvature bound $-(n-1) k(k \geq 0)$, then

$$
\lambda \geq \frac{\exp \left\{-\left[1+\left(1+4(n-1)^{2} d^{2} k\right)^{\frac{1}{2}}\right]\right\}}{(n-1) d^{2}},
$$

where $d=\operatorname{diam}(M), n=\operatorname{dim}(M)$.

For finite connected graphs, Fan Chung [4] got a lower bound for the eigenvalue of Laplace operator, see also Barlow, Coulhon and Grigor'yan [3].

Since for graphs they always have a lower bound on the Ricci curvature, we can expect a similar estimate for the eigenvalue of Laplace operator. In fact we can also get a lower bound similar to the bound of Li and Yau by using the gradient estimate of harmonic eigenfunction on graphs. We expect more results on graphs can be proved in this direction.

Suppose $G$ is a weighted and connected graph (i.e. any two vertices of $G$ can be connected by a path on $G$ ), let $d$ be the weighted degree of $G$, and $D$ be the diameter of $G$. 
Theorem 1.8. Suppose $G$ is a connected graph with diameter $D$, then the nonzero eigenvalue of minus Laplace operator $-\Delta$ on $G$

$$
\lambda \geq \frac{1}{d D(\exp (d D+1)-1)},
$$

Remark 1.9. In [3] and [4], the lower bound for the eigenvalue is $\lambda \geq \frac{1}{D \mathrm{VolG}}$, where $\operatorname{Vol} G$ denote the volume of $G$, given by

$$
\mathrm{Vol} G=\sum_{x} d_{x} .
$$

Suppose $G$ is a d-regular graph(i.e. every vertex of $G$ has same degree $d$ ), in [4] section 6.5 , for given $d$ and the diameter $D$, a graph $G$ can have as many as $d(d-1)^{D}$ vertices, then the $\operatorname{Vol} G=d^{2}(d-1)^{D}$. So in the denominator of the lower bounded of the eigenvalue, we can't expect a polynomial growth in term of diameter $D$. And in most cases, the lower bound of Theorem 1.8 is worse than the lower bound in [3] and [4]. We only get a lower bound similar to the case of manifold by using gradient estimate. By the way, we think the lower bound of Chung can't be improved too much.

\section{The proofs of the Theorem 1.2, Theorem 1.3 and Proposition 1.6}

First we prove the following Lemma. The idea to use the Laplace operator on the gradient is from Yau, Li and others, See [6], [7] and [11]. The notations on the graph are from the book of Chung [4]. Suppose

$$
\rho(x)=|\nabla f|^{2}(x)=\frac{1}{d_{x}} \sum_{(x, y) \in E} \mu_{x y}[f(x)-f(y)]^{2}
$$

then we have

Lemma 2.1. Suppose that $f \in V^{R}$, then for all $x \in V$

$$
\begin{aligned}
\Delta|\nabla f|^{2}(x)= & \frac{1}{d_{x}} \cdot \sum_{(x, y) \in E} \frac{\mu_{x y}}{d_{y}} \sum_{(y, z) \in E} \mu_{y z}[f(x)-2 f(y)+f(z)]^{2}- \\
& \frac{2}{d_{x}} \sum_{(x, y) \in E} \frac{\mu_{x y}}{d_{y}} \sum_{(y, z) \in E} \mu_{y z}[f(x)-2 f(y)+f(z)] \cdot[f(x)-f(y)]
\end{aligned}
$$

Proof. Let

$$
\rho(x)=\frac{1}{d_{x}} \sum_{(x, y) \in E} \mu_{x y}[f(x)-f(y)]^{2},
$$

then

$$
\rho(y)=\frac{1}{d_{y}} \sum_{(y, z) \in E} \mu_{y z}[f(y)-f(z)]^{2}
$$

and 


$$
\begin{aligned}
( & \Delta) \rho(x) \\
= & \frac{1}{d_{x}} \sum_{(x, y) \in E} \mu_{x y}[\rho(x)-\rho(y)] \\
= & \frac{1}{d_{x}}\left[d_{x} \cdot \rho(x)-\sum_{(x, y) \in E} \mu_{x y} \rho(y)\right] \\
= & \frac{1}{d_{x}}\left[d_{x} \cdot \frac{1}{d_{x}} \cdot \sum_{(x, y) \in E} \mu_{x y}(f(x)-f(y))^{2}-\sum_{(x, y) \in E} \frac{\mu_{x y}}{d_{y}} \sum_{(y, z) \in E} \mu_{y z}(f(y)-f(z))^{2}\right] \\
= & \frac{1}{d_{x}}\left[\sum_{(x, y) \in E} \mu_{x y}(f(x)-f(y))^{2}-\sum_{(x, y) \in E} \frac{\mu_{x y}}{d_{y}} \sum_{(y, z) \in E} \mu_{y z}(f(y)-f(z))^{2}\right] \\
= & \frac{1}{d_{x}} \cdot \sum_{(x, y) \in E} \frac{\mu_{x y}}{d_{y}} \sum_{(y, z) \in E} \mu_{y z}\left[(f(x)-f(y))^{2}-(f(y)-f(z))^{2}\right] \\
= & -\frac{1}{d_{x}} \cdot \sum_{(x, y) \in E} \frac{\mu_{x y}}{d_{y}} \sum_{(y, z) \in E} \mu_{y z}[f(x)-2 f(y)+f(z)]^{2}+ \\
& \frac{2}{d_{x}} \sum_{(x, y) \in E} \frac{\mu_{x y}}{d_{y}} \sum_{(y, z) \in E} \mu_{y z}[f(x)-2 f(y)+f(z)] \cdot[f(x)-f(y)] .
\end{aligned}
$$

This proves the Lemma 2.1.

\section{The proof of Theorem 1.2:}

Proof. The Ricci curvature operator

$$
\Gamma_{2}(f, f)=\frac{1}{2}\{\Delta \Gamma(f, f)-2 \Gamma(f, \Delta f)\},
$$

where

$$
\Gamma(f, f)=\frac{1}{2}|\nabla f|^{2} .
$$

The bilinear operator

$$
\Gamma(f, \Delta f)=\frac{1}{2}\left\{\Delta(f \Delta f)-f \Delta(\Delta f)-(\Delta f)^{2}\right\},
$$

where 


$$
\begin{aligned}
\Delta & (f(x) \Delta f(x)) \\
= & \frac{1}{d_{x}} \sum_{(x, y) \in E} \mu_{x y}[f(y) \Delta f(y)-f(x) \Delta f(x)] \\
= & \frac{1}{d_{x}} \sum_{(x, y) \in E} \mu_{x y}[f(y) \Delta f(y)-f(y) \Delta f(x)+f(y) \Delta f(x)-f(x) \Delta f(x)] \\
= & \frac{1}{d_{x}} \sum_{(x, y) \in E} \mu_{x y} f(y)[\Delta f(y)-\Delta f(x)]+\frac{1}{d_{x}} \sum_{(x, y) \in E} \mu_{x y}[f(y)-f(x)] \Delta f(x) \\
= & \frac{1}{d_{x}} \sum_{(x, y) \in E} \mu_{x y}[f(y)-f(x)] \cdot[\Delta f(y)-\Delta f(x)]+ \\
& \frac{1}{d_{x}} \sum_{(x, y) \in E} \mu_{x y} f(x) \cdot[\Delta f(y)-\Delta f(x)]+(\Delta f(x))^{2} \\
= & \frac{1}{d_{x}} \sum_{(x, y) \in E} \mu_{x y}[f(y)-f(x)] \cdot[\Delta f(y)-\Delta f(x)]+f(x) \cdot \Delta(\Delta f(x))+(\Delta f(x))^{2} .
\end{aligned}
$$

Then we have,

$$
\Gamma(f, \Delta f)(x)=\frac{1}{2} \cdot \frac{1}{d_{x}} \sum_{(x, y) \in E} \mu_{x y}[f(y)-f(x)] \cdot[\Delta f(y)-\Delta f(x)] .
$$

The equation (2.2) can also be implied by Lemma 1.4 and polarization. Plugging (2.2) into (2.1) and using Lemma 1.4 and Lemma 2.1, we get

$$
\begin{aligned}
& \Gamma_{2}(f, f)(x) \\
&=\frac{1}{2} \cdot\left\{\Delta\left(\frac{1}{2}|\nabla f|^{2}(x)\right)-\frac{1}{d_{x}} \sum_{(x, y) \in E} \mu_{x y}[f(y)-f(x)] \cdot[\Delta f(y)-\Delta f(x)]\right\} \\
&=\frac{1}{4} \cdot \frac{1}{d_{x}} \cdot \sum_{(x, y) \in E} \frac{\mu_{x y}}{d_{y}} \sum_{(y, z) \in E} \mu_{y z}[f(x)-f(y)-f(y)+f(z)]^{2}- \\
& \\
& \frac{1}{2} \cdot \frac{1}{d_{x}} \sum_{(x, y) \in E} \frac{\mu_{x y}}{d_{y}} \sum_{(y, z) \in E} \mu_{y z}[f(x)-f(y)-f(y)+f(z)] \cdot[f(x)-f(y)]- \\
& \frac{1}{2} \cdot \frac{1}{d_{x}} \sum_{(x, y) \in E} \mu_{x y}[f(y)-f(x)] \cdot\left(\frac{1}{d_{y}} \sum_{(y, z) \in E} \mu_{y z}[f(z)-f(y)]-\right. \\
&\left.\frac{1}{d_{x}} \sum_{(x, y) \in E} \mu_{x y}[f(y)-f(x)]\right)
\end{aligned}
$$




$$
\begin{aligned}
\geq & \frac{1}{4 d_{x}} \cdot \sum_{(x, y) \in E} \frac{\mu_{x y} \mu_{y x}}{d_{y}}[2 f(x)-2 f(y)]^{2}-\frac{1}{2} \cdot \frac{1}{d_{x}} \sum_{(x, y) \in E} \mu_{x y}[f(x)-f(y)]^{2}+ \\
& \frac{1}{2} \cdot \frac{1}{d_{x}} \cdot \sum_{(x, y) \in E} \frac{\mu_{x y}}{d_{y}} \sum_{(y, z) \in E} \mu_{y z}[f(z)-f(y)] \cdot[f(y)-f(x)]- \\
& \frac{1}{2} \cdot \frac{1}{d_{x}} \cdot \sum_{(x, y) \in E} \frac{\mu_{x y}}{d_{y}} \sum_{(y, z) \in E} \mu_{y z}[f(z)-f(y)] \cdot[f(y)-f(x)] \\
& +\frac{1}{2} \cdot\left(\frac{1}{d_{x}} \cdot \sum_{(x, y) \in E} \mu_{x y}[f(y)-f(x)]\right)^{2} \\
\geq & \frac{1}{d} \cdot \frac{1}{d_{x}} \cdot \sum_{(x, y) \in E} \mu_{x y}[f(y)-f(x)]^{2}-\frac{1}{2} \cdot \frac{1}{d_{x}} \cdot \sum_{(x, y) \in E} \mu_{x y}[f(y)-f(x)]^{2} \\
& +\frac{1}{2} \cdot\left(\frac{1}{d_{x}} \cdot \sum_{(x, y) \in E} \mu_{x y}[f(y)-f(x)]\right)^{2} \\
= & \left(\frac{1}{d}+\frac{1}{2}\right) \cdot(\Delta f(x))^{2}-\frac{1}{2}|\nabla f|^{2}(x) \\
= & \frac{1}{2} \cdot(\Delta f(x))^{2}+\left(\frac{1}{d}-1\right) \Gamma(f, f)(x) .
\end{aligned}
$$

where we choose the vertex $z$ that is adjacent to $y$ exactly to be vertex $x$ in the first term of the first inequality, and use $\frac{d_{y}}{\mu_{y x}} \leq d$ in the last inequality.

This proves the Theorem 1.2.

The proof of Theorem 1.3 is similar.

Finally we give the proof of Proposition 1.6: The proof of the first part is similar to the proof of Theorem 1.2 by using a modification of the proof of Lemma 2.1. The idea of the modification comes from the paper [5]. We omit the proof here.

For the proof of the second part of the Proposition 1.6. Suppose the triangle graph $G$ with three vertices $x, y$ and $z$. It is easy to imply the following equalities by definition:

$$
\Delta\left(\frac{1}{2}|\nabla f|^{2}(x)\right)=\frac{1}{8}\left[2(f(z)-f(y))^{2}-(f(y)-f(x))^{2}-(f(z)-f(x))^{2}\right]
$$

and

$$
\Gamma(f, \Delta f)=-\frac{3}{8}\left[(f(y)-f(x))^{2}+(f(z)-f(x))^{2}\right] .
$$

Therefore we have: 
RICCI CURVATURE ON GRAPHS

$$
\begin{aligned}
\Gamma_{2} & (f, f)(x) \\
& =\frac{1}{2} \cdot\left\{\Delta\left(\frac{1}{2}|\nabla f|^{2}(x)\right)-2 \Gamma(f, \Delta f)\right\} \\
& =\frac{1}{16} \cdot\left[2(f(z)-f(y))^{2}+5(f(y)-f(x))^{2}+5(f(z)-f(x))^{2}\right] \\
& \geq \frac{1}{16} \cdot\left[4(f(y)-f(x))^{2}+4(f(z)-f(x))^{2}+(f(y)-f(x))^{2}+(f(z)-f(x))^{2}\right] \\
\geq & \frac{1}{16} \cdot\left[2(f(y)-f(x)+f(z)-f(x))^{2}+(f(y)-f(x))^{2}+(f(z)-f(x))^{2}\right] \\
& =\frac{1}{2} \cdot(\Delta f(x))^{2}+\frac{1}{4} \Gamma(f, f)(x)
\end{aligned}
$$

This finish the proof of Proposition 1.6.

\section{The Proof of Theorem 1.8}

In this section, we will prove the Theorem 1.8.

Suppose $f$ is a harmonic eigenfunction of $G$ with eigenvalue $\lambda \neq 0$, then

$$
\begin{aligned}
\sum_{x \in V} d_{x} f(x) & =\frac{1}{\lambda} \sum_{x \in V} d_{x}(-\Delta) f(x) \\
& =\frac{1}{\lambda} \sum_{x \in V} \sum_{(x, y) \in E} \mu_{x y}[f(x)-f(y)] \\
& =\frac{1}{\lambda} \sum_{x \in V}\left[d_{x} f(x)-\sum_{(x, y) \in E} \mu_{x y} f(y)\right] \\
& =0
\end{aligned}
$$

So we can assume that

$$
\sup f=1>\inf f=k,
$$

where $k<0$. We can also assume that $k>-1$.

First we prove the following gradient estimate for the harmonic eigenfunction $f$.

Lemma 3.1. Suppose $G$ is a locally finite graph, then

$$
\frac{|\nabla f|(x)}{(\beta-f(x))} \leq \sqrt{d}\left(\frac{1}{\beta-1} \lambda+1\right)
$$

where $\beta>1$.

Proof. Since $f$ is a harmonic eigenfunction of $G$ with eigenvalue $\lambda \neq 0$, then

$$
\begin{aligned}
(-\Delta) f(x) & =\frac{1}{d_{x}} \sum_{(x, y) \in E} \mu_{x y}[f(x)-f(y)] \\
& =\lambda f(x)
\end{aligned}
$$

This implies that

$$
\frac{1}{d_{x}} \sum_{(x, y) \in E} \mu_{x y} f(y)=(1-\lambda) f(x) .
$$

Then we have 


$$
\begin{aligned}
& \frac{|\nabla f|^{2}(x)}{(\beta-f(x))^{2}} \\
& =\frac{\frac{1}{d_{x}} \sum_{(x, y) \in E} \mu_{x y}[f(x)-f(y)]^{2}}{(\beta-f(x))^{2}} \\
& =\frac{\sum_{(x, y) \in E} \mu_{x y}[(\beta-f(x))-(\beta-f(y))]^{2}}{d_{x}(\beta-f(x))^{2}} \\
& =\frac{\sum_{(x, y) \in E} \mu_{x y}(\beta-f(x))^{2}-2 \sum_{(x, y) \in E} \mu_{x y}(\beta-f(x))(\beta-f(y))}{d_{x}(\beta-f(x))^{2}} \\
& +\frac{\sum_{(x, y) \in E} \frac{1}{\mu_{x y}} \mu_{x y}^{2}(\beta-f(y))^{2}}{d_{x}(\beta-f(x))^{2}} \\
& \leq \frac{\sum_{(x, y) \in E} \mu_{x y}(\beta-f(x))^{2}-2 \sum_{(x, y) \in E} \mu_{x y}(\beta-f(x))(\beta-f(y))}{d_{x}(\beta-f(x))^{2}} \\
& +\frac{\frac{d}{d_{x}}\left(\sum_{(x, y) \in E} \mu_{x y}(\beta-f(y))\right)^{2}}{d_{x}(\beta-f(x))^{2}} \\
& =\frac{d_{x}(\beta-f(x))^{2}-2 d_{x}(\beta-f(x))(\beta-(1-\lambda) f(x))+d d_{x}(\beta-(1-\lambda) f(x))^{2}}{d_{x}(\beta-f(x))^{2}} \\
& =\frac{(\beta-f(x))^{2}-2(\beta-f(x))(\beta-f(x)+\lambda f(x))+d(\beta-f(x)+\lambda f(x))^{2}}{(\beta-f(x))^{2}} \\
& =\frac{-(\beta-f(x))^{2}+2(d-1)(\beta-f(x)) \lambda f(x)+d(\beta-f(x))^{2}+d \lambda^{2} f^{2}(x)}{(\beta-f(x))^{2}} \\
& =d-1+2(d-1) \frac{\lambda f(x)}{(\beta-f(x))}+d \frac{\lambda^{2} f(x)^{2}}{(\beta-f(x))^{2}} \\
& \leq d-1+2(d-1) \frac{\lambda}{(\beta-1)}+d \frac{\lambda^{2}}{(\beta-1)^{2}} \\
& \leq d\left(\frac{1}{\beta-1} \lambda+1\right)^{2} \text {. }
\end{aligned}
$$

In the proof of the first inequality, we use the fact that $\mu_{x y}(\beta-f(y))>0$ and $\frac{d_{x}}{\mu_{x y}} \leq d$, then the sum of square of positive numbers is less than the square of the sum of positive numbers. In the proof of the second inequality, we use the fact that $d \geq 1$ and $f(x) \leq 1$.

From this we prove the Lemma 3.1.

\section{The proof of Theorem 1.8:}

Proof. Take $x_{1}, x_{n}$ in $G$, such that $f\left(x_{1}\right)=\sup f=1, f\left(x_{n}\right)=\inf f=k<0$ and let $x_{1}, x_{2}, \ldots x_{n}$ be a shortest path connecting $x_{1}$ and $x_{n}$, where $\left(x_{i}, x_{i+1}\right) \in E$. Then $n \leq D$. 
From the Lemma 3.1, we have

$$
\frac{\left(\frac{1}{d_{x}} \sum_{(x, y) \in E} \mu_{x y}[f(x)-f(y)]^{2}\right)^{\frac{1}{2}}}{(\beta-f(x))} \leq \sqrt{d}\left(\frac{1}{\beta-1} \lambda+1\right) .
$$

Since

$$
\frac{\left|f\left(x_{i}\right)-f\left(x_{i+1}\right)\right|}{\beta-f\left(x_{i}\right)} \leq \frac{\left(\frac{1}{d_{x_{i}}} \sum_{\left(x_{i}, y_{i}\right) \in E} \frac{d_{x_{i}}}{\mu_{x_{i} y_{i}}} \mu_{x_{i} y_{i}}\left[f\left(x_{i}\right)-f\left(y_{i}\right)\right]^{2}\right)^{\frac{1}{2}}}{\left(\beta-f\left(x_{i}\right)\right)} \leq \sqrt{d} \frac{|\nabla f|\left(x_{i}\right)}{\left(\beta-f\left(x_{i}\right)\right)},
$$

we get

$$
\sum_{i=1}^{n-1} \frac{\left|f\left(x_{i}\right)-f\left(x_{i+1}\right)\right|}{\beta-f\left(x_{i}\right)} \leq \operatorname{Dd}\left(\frac{1}{\beta-1} \lambda+1\right)
$$

The left term side

$$
\begin{aligned}
& \sum_{i=1}^{n-1} \frac{\left|f\left(x_{i}\right)-f\left(x_{i+1}\right)\right|}{\beta-f\left(x_{i}\right)} \\
\geq & \sum_{i=1}^{n-1} \log \left(1+\frac{\left|f\left(x_{i}\right)-f\left(x_{i+1}\right)\right|}{\beta-f\left(x_{i}\right)}\right) \\
\geq & \sum_{i=1}^{n-1} \log \frac{\beta-f\left(x_{i}\right)+f\left(x_{i}\right)-f\left(x_{i+1}\right)}{\beta-f\left(x_{i}\right)} \\
= & \sum_{i=1}^{n-1} \log \frac{\beta-f\left(x_{i+1}\right)}{\beta-f\left(x_{i}\right)} \\
\geq & \log \frac{\beta}{\beta-1}
\end{aligned}
$$

therefore,

$$
\lambda \geq(\beta-1)\left(\frac{1}{d D} \log \frac{\beta}{\beta-1}-1\right)
$$

To choose the $\beta$ from the identity

$$
\frac{1}{d D} \log \frac{\beta}{\beta-1}-1=\frac{1}{d D},
$$

we get

$$
\lambda \geq \frac{1}{d D(\exp (d D+1)-1)},
$$

This proves the Theorem 1.8.

\section{Acknowledgements}

The authors thank the referee for many nice comments. The first author also like to thank Liu Yuan for some discussions. 


\section{References}

[1] D. Bakry and M. Emery, Diffusions hypercontractives , Sem. de Prob.XIX, Lecture Notes in Math. 1123(1985) 177-206 Springer-verlag.

[2] D. Bakry, M. Ledoux, Z. Qian, Logarithmic Sobolev inequalities, Poincare inequalities and heat kernel bounds. unpublished manuscript.

[3] M. Barlow, T. Coulhon and A. Grigor'yan, Manifolds and graphs with slow heat kernel decay, Inventions Mathematicae, 144(2001),No.3, 609-649.

[4] Fan R. K., Chung, Spectral Graph Theory, CBMS Regional Conference Series in Mathematics, 1997, Number 92, American Mathematical Society.

[5] Fan R. K., Chung and S. T. Yau, Logarithnic Harnack inequalities, Mathematics Research Letters, 3 (1996), 793-812.

[6] P. Li and S. T. Yau, Estimates of eigenvalues of a compactRiemanian manifold, AMS Symposium on the Geometry of the Laplace Operator, University of Hawaii at Manoa, 1979, 205-239.

[7] P. Li and S. T. Yau, On the parabolic kernel of the Schrodinger operator, Acta Math. 156(1986), no. 3-4, 153-201.

[8] J. Lott and C. Villani, Ricci curvature for metric-measure spaces via optimal transport, Annals of Mathematics, 169(2009), 903-991.

[9] S. Ohta, On measure contraction property of metric measure spaces, Comment. Math. Helv. 82(2007), 805-828

[10] Y. Ollivier, Ricci curvature of Markov chains on metric spaces, J. Funct. Anal. 256(2009), no. $3,810-864$.

[11] R. Schoen and S. T. Yau, Lecture on Differential Geometry, International Press Incorporated, Boston, 1994.

[12] K.-T. Sturm, On the geometry of metric measure spaces, (I),(II), Acta Math. 196(2006), no. 1, 65-131, 133-177.

Department of Mathematics, Information School, Renmin University of China, Beijing, 100872,CHINA

E-mail address: linyong01@ruc.edu.cn

Department of Mathematics, Harvard University, One Oxford Street, Cambridge, Ma 02138, USA.

E-mail address: yau@math.harvard.edu 